\title{
The connection between elastic scattering cross sections and acoustic vibrations of an embedded nanoparticle
}

\author{
L. Saviot ${ }^{* 1}$ and D. B. Murray ${ }^{2}$ \\ ${ }^{1}$ Laboratoire de Recherche sur la Réactivité des Solides, UMR 5613 CNRS - Université de Bourgogne, \\ Dijon, France \\ ${ }^{2}$ Department of Physics and Astronomy, Okanagan University College, Kelowna, British Columbia, \\ Canada V1V 1V7
}

\begin{abstract}
Arbitrary waves incident on a solid embedded nanoparticle are studied. The acoustic vibrational frequencies are shown to correspond to the poles of the scattering cross section in the complex frequency plane. The location of the poles is unchanged even if the incident wave is nonplanar. A second approach approximating the infinite matrix as a very large shell surrounding the nanoparticle provides an alternate way of predicting the mode frequencies. The wave function of the vibration is also provided.
\end{abstract}

1 Introduction In the last two decades, a significant number of works have been devoted to the study of acoustic vibrations of spherical nanoparticles. This has aided the understanding of the role of vibrations in the performance of some optical devices (for example, in electronic dephasing due to emission of phonons). These vibrations can be experimentally investigated with different techniques including Raman scattering[1], photoluminescence[2], and femtosecond pump-probe experiments[3]. Usually, continuum elastic models have been used to describe these vibrations. The original 1882 work by Lamb [4] found the vibrational frequencies of a free continuous isotropic elastic sphere. However in real life, nanoparticles are seldom free but rather embedded in another medium such as a glass matrix or liquid.

The problem of eigenmodes of vibrations of an embedded sphere has not been extensively studied. However, a lot of work has been devoted to the apparently unrelated problem of scattering of acoustic waves by a sphere[5]. That is where Dubrovskiy et al. paper[6] has its starting point to calculate complex natural frequencies of vibrations of an embedded sphere. Although this method, referred to here as the complex frequency model (CFM), is a very powerful tool, one might wonder about the significance of the complex frequencies and the relevance of discrete natural vibrations in a problem where one expects a continuum of vibrational state. The goals of this paper are to explain the basics of this model and to show its relevance by comparing it with the core shell model (CSM) [7] which is a different approach using real valued frequencies.

2 Scattering of elastic waves and CFM The usual basis of unit vectors in spherical coordinates is ( $\hat{\mathbf{e}}_{r}$, $\left.\hat{\mathbf{e}}_{\theta}, \hat{\mathbf{e}}_{\phi}\right)$. However, it is more convenient to express results using a different set of vectors. In this paper, we will use basic vectors $\left(\boldsymbol{e}_{1}, \boldsymbol{e}_{2}, \boldsymbol{e}_{3}\right)$ defined as follows:

$$
\begin{aligned}
\boldsymbol{e}_{1} & =Y_{\ell}^{m} \hat{\mathbf{e}}_{r} \\
\boldsymbol{e}_{2} & =\frac{1}{\sin \theta} \frac{\partial Y_{\ell}^{m}}{\partial \phi} \hat{\mathbf{e}}_{\phi}+\frac{\partial Y_{\ell}^{m}}{\partial \theta} \hat{\mathbf{e}}_{\theta} \\
\boldsymbol{e}_{3} & =\frac{1}{\sin \theta} \frac{\partial Y_{\ell}^{m}}{\partial \phi} \hat{\mathbf{e}}_{\theta}-\frac{\partial Y_{\ell}^{m}}{\partial \theta} \hat{\mathbf{e}}_{\phi}
\end{aligned}
$$

\footnotetext{
* Corresponding author: e-mail: lucien.saviot@u-bourgogne.fr
} 
where $Y_{\ell}^{m}$ represents the spherical harmonics.

Vibrational modes of a sphere, whether free or embedded, may be classified as "torsional" (TOR) or "spheroidal" (SPH). Torsional modes are those for which the displacement field has zero divergence. All other modes are called spheroidal. Torsional modes have non-zero displacement along $\boldsymbol{e}_{3}$ only. Spheroidal modes have non-zero displacement along $\boldsymbol{e}_{1}$ and $\boldsymbol{e}_{2}$ only. For $\ell=0$, we have $\boldsymbol{e}_{2}=\boldsymbol{e}_{3}=\mathbf{0}$.

2.1 Basic equations In order to clarify the meaning of the complex-valued eigenfrequencies of an embedded elastic sphere, the problem we want to investigate is the scattering of an incident elastic wave in a medium (matrix) by a sphere made of a different material than the matrix. Three different terms have to be considered: the incident wave, the motion inside the sphere and the scattered wave. All these waves have to satisfy the following wave equation (assuming an $\exp (-i \omega t)$ time dependence) inside the medium where they exist.

$$
(\lambda+2 \mu) \nabla(\nabla \cdot \boldsymbol{u})-\mu \nabla \times(\nabla \times \boldsymbol{u})+\rho \omega^{2} \boldsymbol{u}=0
$$

$\boldsymbol{u}$ is the displacement, $\lambda$ and $\mu$ the Lamé coefficients and $\rho$ the mass density.

Thus, they can be decomposed into the the following form:

$$
\boldsymbol{u}=\nabla \Phi+\nabla \times(\boldsymbol{r} \chi+\nabla \times(\boldsymbol{r} \psi))
$$

where the three potential fields are

$$
\begin{aligned}
\Phi & =\sum_{\ell=0, m} A_{\ell m} z_{\ell}\left(k_{L} r\right) Y_{\ell}^{m}(\theta, \phi) \\
\chi & =\sum_{\ell=1, m} B_{\ell m} z_{\ell}\left(k_{T} r\right) Y_{\ell}^{m}(\theta, \phi) \\
\psi & =\sum_{\ell=1, m} C_{\ell m} z_{\ell}\left(k_{T} r\right) Y_{\ell}^{m}(\theta, \phi)
\end{aligned}
$$

$z_{\ell}$ is the relevant spherical Bessel function of order $\ell$, (first or second kind or Hankel) $k_{L}$ and $k_{T}$ are longitudinal and transverse wavevectors respectively.

2.2 Solutions Using the notations introduced by Eringen et al. [8], the displacement and the associated surface traction (surface force per unit area) $\boldsymbol{T}=\sigma_{r r} \hat{\mathbf{e}}_{r}+\sigma_{r \theta} \hat{\mathbf{e}}_{\theta}+\sigma_{r \phi} \hat{\mathbf{e}}_{\phi}$ of the $(\ell, m)$ component can be written as follows:

\begin{tabular}{c|c|c} 
potential & $\boldsymbol{u}$ & $\boldsymbol{T}$ \\
\hline$\Phi$ & $U_{1}^{z}\left(\ell, k_{L} r\right) \boldsymbol{e}_{1}+U_{3}^{z}\left(\ell, k_{L} r\right) \boldsymbol{e}_{2}$ & $T_{11}^{z}\left(\ell, k_{L} r\right) \boldsymbol{e}_{1}+T_{13}^{z}\left(\ell, k_{L} r\right) \boldsymbol{e}_{2}$ \\
$\chi$ & $V_{2}^{z}\left(\ell, k_{T} r\right) \boldsymbol{e}_{3}$ & $T_{42}^{z}\left(\ell, k_{T} r\right) \boldsymbol{e}_{3}$ \\
$\psi$ & $V_{1}^{z}\left(\ell, k_{T} r\right) \boldsymbol{e}_{1}+V_{3}^{z}\left(\ell, k_{T} r\right) \boldsymbol{e}_{2}$ & $T_{41}^{z}\left(\ell, k_{T} r\right) \boldsymbol{e}_{1}+T_{43}^{z}\left(\ell, k_{T} r\right) \boldsymbol{e}_{2}$
\end{tabular}

The $z$ superscript has been added to recall the nature of the spherical Bessel function to use. Inside the sphere, $z$ is the spherical Bessel function of the first kind $j$. Outside the sphere, in the general case, two terms have to be considered where $z$ is $j$ and $n$ (spherical Bessel function of the second kind). For the case of the outgoing scattered wave, we'll use the spherical Hankel function of the first kind $h_{\ell}^{(1)}(x)=$ $j_{\ell}(x)+i n_{\ell}(x)$. 
2.3 Boundary conditions The boundary conditions are the usual continuities of displacement and surface traction at the sphere-matrix interface. Because the boundary conditions should be satisfied for $r=R$ (sphere radius) at all $\theta$ and $\phi$, each equation of continuity breaks up into a linear inhomogeneous system of six equations for each $(\ell, m)$ the incident wave is decomposed onto. The unknowns are the amplitudes of the vibration inside the sphere and of the scattered wave. The constants are the amplitudes of the decomposition of the incident wave on $(\ell, m)$. Therefore the unknowns can be expressed as the ratio of two $6 \times 6$ determinants. The determinant equation in the denominator is the one of the homogeneous system, i. $e$. for $\ell>0$ and $|m| \leq \ell$ :

$$
D_{\ell}=\left|\begin{array}{cccccc}
U_{1}^{j}(\ell, \xi) & U_{3}^{j}(\ell, \eta) & U_{1}^{h^{(1)}}(\ell, \xi) & U_{3}^{h^{(1)}}(\ell, \eta) & 0 & 0 \\
V_{1}^{j}(\ell, \xi) & V_{3}^{j}(\ell, \eta) & V_{1}^{h^{(1)}}(\ell, \xi) & V_{3}^{h^{(1)}}(\ell, \eta) & 0 & 0 \\
\mu_{p} T_{11}^{j}(\ell, \xi) & \mu_{p} T_{13}^{j}(\ell, \eta) & \mu_{m} T_{11}^{h^{(1)}}(\ell, \xi) & \mu_{m} T_{13}^{h^{(1)}}(\ell, \eta) & 0 & 0 \\
\mu_{p} T_{41}^{j}(\ell, \xi) & \mu_{p} T_{43}^{j}(\ell, \eta) & \mu_{m} T_{41}^{h^{(1)}}(\ell, \xi) & \mu_{m} T_{43}^{h^{(1)}}(\ell, \eta) & 0 & 0 \\
0 & 0 & 0 & 0 & V_{2}^{j}(\ell, \eta) & V_{2}^{h^{(1)}}(\ell, \eta) \\
0 & 0 & 0 & 0 & \mu_{p} T_{42}^{j}(\ell, \eta) & \mu_{m} T_{42}^{h^{(1)}}(\ell, \eta)
\end{array}\right|
$$

where $\xi=k_{L} R, \eta=k_{T} R, \mu_{p}$ and $\mu_{m}$ are the particle and matrix mass densities and the rows represent the continuity of the displacement along $\boldsymbol{u} \cdot \boldsymbol{e}_{1}, \boldsymbol{u} \cdot \boldsymbol{e}_{2}, \boldsymbol{T} \cdot \boldsymbol{e}_{1}, \boldsymbol{T} \cdot \boldsymbol{e}_{2}, \boldsymbol{u} \cdot \boldsymbol{e}_{3}$ and $\boldsymbol{T} \cdot \boldsymbol{e}_{3}$ from top to bottom. The columns represent the coefficients for $\Phi_{p}, \psi_{p}, \Phi_{m}, \psi_{m}, \chi_{p}$, and $\chi_{m}$ where the $p$ and $m$ subscripts are for waves inside the particle and the outgoing wave inside the matrix respectively.

Since the matrix is block diagonal this determinant is easily decomposed as the product of $\Delta_{\ell}(4 \times 4$ determinant for SPH modes $)$ and $\Gamma_{\ell}(2 \times 2$ determinant for TOR modes $)$.

For $\ell=0$, both $\boldsymbol{e}_{2}$ and $\boldsymbol{e}_{3}$ equal $\mathbf{0}$ and the determinant is:

$$
D_{0}=\Delta_{0}=\left|\begin{array}{cc}
U_{1}^{j}(\ell=0, \xi) & U_{1}^{h^{(1)}}(\ell=0, \xi) \\
\mu_{p} T_{11}^{j}(\ell=0, \xi) & \mu_{m} T_{11}^{h^{(1)}}(\ell=0, \xi)
\end{array}\right|
$$

These determinants do not depend on the azimuthal quantum number $m$. They are also independent of the nature of the incident wave. This makes the results equally applicable to a situation where the incident wave is a spherical wave emanating from a source close by to the scattering sphere. This would be the case in a phononic crystal, where elastic waves scatter sequentially from a periodic array of scatterers.

2.4 CFM Up to now, only real valued $\omega$ have been considered. The amplitudes of the wave inside the sphere and of the outgoing wave have been shown to be the ratio of two determinants with the same determinant in the denominator (the determinant of the homogeneous linear system). These amplitudes can therefore become quite large when this determinant is close to zero. The physical system corresponding to the system of homogeneous equations is the one where there is no incident wave. In this case, the only solution is obtained when all the amplitudes are zero. Therefore $D_{\ell}$ does not vanish for real values of $\omega$. However, it can reach local minima. In order to predict the position of these minima, we are going to locate the complex roots of the matrix determinant $D_{\ell}$. This is the heart of the complex frequency model introduced by Dubrovskiy and Morochnik[6]. Because of the $\exp (-i \omega t)$ dependence of the displacement, the real parts of the CFM roots correspond to the position of the resonance and the imaginary parts correspond to its width and its damping. Of course, this interpretation is just an approximation and we can't expect it to precisely reproduce the position and width of the embedded sphere resonances in every case. As seen before, $\Delta_{l}$ does not depend on the nature of the incident wave. Therefore its roots can be associated with the embedded sphere natural vibrations which we prefer to term pseudo-modes of vibrations.

Two kinds of CFM roots exist. Some reflect primarily natural vibrations of the sphere. Some others are much more damped and reflect primarily the vibrations of the matrix around the sphere. The latter (later 
referred to as "matrix modes") appear as a background in the scattering calculations. Sphere vibrations are narrower peaks above this background. Of course, this distinction becomes harder to make when the sphere and the matrix have similar characteristics (mass density and sound velocities). Therefore, there are several limitations inherent to the CFM model:

1. it is an approximation (because knowing the complex roots of $\Delta_{l}$ is not enough to know its variation for real arguments)

2. what's the relative importance of sphere and matrix modes?

3. this approach does not provide valid wave functions which are required for Raman scattering spectra calculations for example

3 CSM To overcome the limitations of the CFM model, it is necessary to use a different approach. Instead of starting from a formulation originating from an elastic wave scattering problem, it seems more interesting to focus on the specificities of optical properties of nanoparticles. In the Raman scattering problem, the vibration eigenmodes and eigenenergies of the system are important. Then, for a given eigenmode, the Raman intensity is related to the displacement inside the particle (in most usual cases, Raman scattering from the matrix is negligible in this energy range). In a recent paper[7], we modeled the matrix embedded nanoparticle by a core-shell system where the "core" is the nanoparticle and the "shell" is the matrix. The shell's external radius is chosen several orders of magnitude bigger than the nanoparticle radius. The mean squared displacement within the nanoparticle interior $\left(\left\langle u^{2}\right\rangle_{p}\right)$ was monitored by integrating the squared displacement field over the interior of the core as a function of the eigenmode energy. This uses displacement fields that were normalized over the entire core and matrix. As was shown, very good agreement is obtained between the frequency and width of particle modes calculated with CFM and peaks in the plots of $\left\langle u^{2}\right\rangle_{p}$ as a function of the mode frequency. The validity of the CFM model is challenged when the nanoparticle and the matrix are made of materials having similar mass densities and sound velocities, for example for a silicon nanoparticle inside silica. In such cases, the CFM particle modes are still in good agreement with the peaks in the $\left\langle u^{2}\right\rangle_{p}$ plots, but a significant background is seen below these peaks.

4 Conclusion Depending on the precision required for a given task, there are several ways one can approach calculations of natural vibrations of a free or embedded elastic sphere. If the sphere is free, then the Lamb model suffices. When the sphere is not free but only the frequency of the natural vibrations matters, the Lamb model (free sphere model (FSM)) or the bound sphere model[7] (BSM) will suffice in most cases as a workable approximation. When the width of the resonance matters the CFM is an accurate tool. CFM also fills in a few special cases not addressed by FSM, such as the existence of matrix modes. Finally, for situations such as the calculation of the absolute intensity or shape of low-frequency Raman spectra, models such as the CSM are necessary.

\section{References}

[1] E. Duval, A. Boukenter, and B. Champagnon. Phys. Rev. Lett 56, 2052 (1986)

[2] P. Palinginis, S. Tavenner, M. Lonergan, and H. Wang. Phys. Rev. B 67, 201307 (2003)

[3] N. Del Fatti, C. Voisin, F. Chevy, F. Vallée, and C. Flytzanis. J. Chem. Phys. 110, 11484 (1999)

[4] H. Lamb. Proc. London Math. Soc. 13, 189 (1882)

[5] D. Brill and G. Gaunaurd. J. Acoust. Soc. Am. 81, 1 (1987) See also references therein.

[6] V. A. Dubrovskiy and V. S. Morochnik. Earth Physics 17, 494 (1981)

[7] D. B. Murray and L. Saviot. Phys. Rev. B 69, 094305 (2004) cond-mat/0310099

[8] A. C. Eringen and E. S. Suhubi. Elastodynamics, volume II, 804-833. Academic, New York, 1975. 